\title{
Autorité scientifique et épistémique à l'épreuve de la mesure des citations
}

Scientific and Epistemic Authority and Citation Metrics

\section{Évelyne Broudoux}

\section{(2) OpenEdition}

\section{Journals}

Édition électronique

URL : http://journals.openedition.org/edc/6841

DOI : 10.4000/edc. 6841

ISSN : 2101-0366

Éditeur

Université Lille-3

Édition imprimée

Date de publication : 1 juin 2017

Pagination : 177-198

ISBN : 978-2-917562-17-8

ISSN : $1270-6841$

Référence électronique

Évelyne Broudoux, «Autorité scientifique et épistémique à l'épreuve de la mesure des citations », Études de communication [En ligne], 48 | 2017, mis en ligne le 01 juin 2017, consulté le 19 avril 2019 URL : http://journals.openedition.org/edc/6841 ; DOI : 10.4000/edc.6841 


\section{Autorité scientifique et épistémique à l'épreuve de la mesure des citations \\ Scientific and Epistemic Authority and Citation Metrics}

Évelyne Broudoux

INTD - CNAM,

Laboratoire DICEN-IDF

evelyne.broudoux@cnam.fr 
Cet article fait le point sur la construction de l'autorité scientifique dans la communication scientifique en examinant sous l'angle du repérage des autorités épistémique et scientifique :

- le champ social des disciplines scientifiques,

- les études réalisées autour de l'analyse des citations et ses mesures,

- I'usage croissant du web social et l'auto-référence,

- la sémantisation des citations.

L'objectif étant de regarder les enjeux actualisés de la communication scientifique.

Mots-clés : analyse de citation, métrique, altmetrics, facteur d'impact, autorité scientifique, autorité épistémique, communication scientifique.
This article explores the construction of scientific and epistemic authority in scholarly communication. It provides an overview of :

- academic disciplines as social fields,

- studies on citation analysis and citation metrics,

- the increasing use, in academic communities, of the social web and self-referencing,

- the semanticizing of citations.

The aim of this article is to identify emerging challenges in the domain of scholarly communication.

Keywords: citation analysis, metrics, altmetrics, impact factor, scientific authority, epistemic authority, scholarly communication. 
Le domaine de la communication scientifique se modifiant à l'heure des nouveaux médias, nous proposons ici une étude préalable examinant une forme particulière d'autorité, celle que nous nommons autorité scientifique. Il s'agirait de détecter les modifications de la mesure de l'influence dans les processus de construction d'autorité afin d'identifier les éventuels "nouveaux " biais à l'œuvre dans les étapes d'évaluation des publications scientifiques. En effet, dans la communication scientifique, il existe aujourd'hui de nombreux moyens de médiatiser les recherches en train de se faire avant même le moment de publier des résultats. Nous nous intéresserons donc aux méthodes de classement de la production de recherche publiée qui sert aujourd'hui à l'évaluation des chercheurs et de leurs équipes.

La démarche adoptée consiste à considérer les approches empruntées à l'histoire et à la sociologie des sciences, à la sociologie et aux sciences de I'information et de la communication. II s'agit aussi de positionner les pratiques actuelles de médiatisation des résultats des recherches scientifiques sur une échelle de temps longue pour mieux apprécier les phénomènes actuels de modification de la communication scientifique sur le web.

Pour commencer, nous examinerons les agencements entre autorité épistémique et scientifique, entre champ et discipline. L'histoire et la sociologie des sciences ont été les disciplines premières à s'intéresser à la fabrication des faits scientifiques. Resituer historiquement les phénomènes d'attribution ou de dénégation d'autorité scientifique est une première entrée nous permettant d'observer les liens noués entre autorité scientifique et autorité épistémique. Nous revisitons ensuite la sociologie du champ scientifique qui a rendu visible la lutte des chercheurs pour les places dominantes au sein des communautés académiques.

Le fait citationnel est constitutif du texte et son analyse est complexe comme l'ont montré les théories littéraires et sémiotiques de l'intertextualité que nous n'aborderons pas ici. Les études quantitatives qui analysent les citations selon des critères d'influence constituent le périmètre de l'approche retenue pour cet article. La présentation en mode chronologique permet de faire ressortir plusieurs périodes : après le démarrage des index de citations suivent des études interrogeant la compréhension de l'activité citationnelle elle-même, et les motivations des chercheurs à référencer d'autres auteurs.

L'analyse de l'évolution des facteurs d'impacts et de leur adoption, leur diversification ou leur abandon par les éditeurs dans le classement de leurs revues indique qu'une réorientation est en train de s'opérer.

La dernière partie fait le constat du déplacement de curseur de l'évaluation du cœur de la production scientifique vers les échanges qu'elle suscite dans le web social. 


\section{1. \\ Autorité scientifique et épistémique, champ et discipline}

Afin de circonscrire cette étude, nous délimitons son espace sémantique : nous distinguons ici l'autorité scientifique de l'autorité épistémique et le champ de la discipline.

L'autorité épistémique concerne l'expertise des savoirs, elle s'intéresse aux connaissances produites, leur organisation et leur représentation. L'étude des processus de construction des savoirs se compose de travaux sur leur conception, leur légitimation, leur réception et leur critique. L'autorité scientifique est liée aux personnes - individu et collectif - agissantes dans l'espace épistémique peuplé par les connaissances scientifiques disciplinaires. La spécificité de l'autorité scientifique est qu'elle se bâtit dans la communauté des pairs où les connaissances sont reconnues comme fiables lorsque les énoncés échangés ont été passés au crible de l'évaluation par les pairs et de la transmission disciplinaire. La discipline repose sur un système structuré de connaissances mettant en œuvre des épistémologies, des concepts, des méthodes, des terrains d'expérimentation et d'observation qui lui sont propres. Elle possède une identité académique constituée par des instances institutionnelles qui en délimitent son périmètre. Le champ est constitué par les espaces sur lesquels s'exerce l'action scientifique (calculs, expérimentations, terrains d'observations) à partir desquels se construisent les disciplines. Mais le champ est aussi " social » et cette caractéristique surdétermine des enjeux échappant aux autorités épistémiques.

Dans un premier temps, nous pouvons dire que les travaux scientifiques faisant autorité sont ceux reconnus comme majeurs pour l'avancement et le développement des disciplines ; toutefois l'histoire prouve que cette autorité est déniée ou tarde à être reconnue lors des changements de paradigme scientifique (Bachelard, 1934) puis (Kuhn, 1962), car les bouleversements dans les théories scientifiques remettent en question ce qui fait tenir ensemble les « collectifs de pensée " au sens de Fleck (1935). En effet, en étudiant la construction du concept de syphilis sur cinq siècles, le bactériologiste Fleck a montré que l'orientation des recherches est conditionnée par des "systèmes d'opinions »"

1 Fleck définit le système d'opinions comme un état des connaissances stable, exigeant de fonctionner comme une totalité fermée, pour perdurer dans le temps long de telle sorte que :

«1. Une remise en cause du système apparait impossible.

2. Ce qui ne se conforme pas au système reste invisible ou.

3. Est passé sous silence même si cela est connu, ou encore.

4. Est déclaré, au prix d'efforts considérables, comme n'étant pas en contradiction avec le système.

5. On voit, décrit et représente des états des choses qui correspondent aux vues dominantes, c'est-à-dire qu'ils en sont pour ainsi dire des réalisations sans que les droits des opinions opposées soient pris en considération (Fleck, 2008, 56). 
qui ne retiennent sur l'ensemble des faits présents que les éléments qui ne remettent pas en question la pertinence desdits systèmes.

Depuis, la sociologie des sciences a pris le relais de cet " établissement des faits » avec la déconstruction du « laboratoire » opérée par Latour et Woolgar qui ont mis en perspective, en se référant à la phénoménotechnique de Bachelard, le rôle des inscriptions produits par les appareils dans la construction du sens des objets observés.

Cette socialité du travail de recherche a également été objectivée par le travail de Pontille (2006) sur l'auctorialité scientifique. Il rappelle la dimension collective du régime d'attribution scientifique qui s'oppose à la conception littéraire et juridique de l'auteur ayant fait du sujet énonciateur une entité individuelle indépendante. La signature de l'auteur scientifique est modelée par sa communauté d'appartenance, et ceci est particulièrement vérifié dans des disciplines comme le biomédical où plus l'article comporte de signatures, moins sa pertinence est à démontrer. C'est alors le « collectif qui reconduit en permanence la distribution circulaire des compétences, des qualités et des attributs des personnes dans la composition de l'auteur » (Pontille, 2006, 13).

Pour aller plus loin dans la découverte de la socialité des pratiques scientifiques liées aux publications, nous nous saisissons du concept de " champ social » qui, bien que ne s'adaptant pas à tous les univers sociaux (Lahire, 2001), a le mérite d'avoir fait ses preuves.

Le champ scientifique a pour spécificité de se dérouler devant les " pairs-concurrents » qui, aptes à reconnaître la valeur des propositions, sont aussi les plus ardus à leur critique. Pour Bourdieu (1976), l'enjeu spécifique est le monopole de l'autorité scientifique qui se compose autant de la « capacité technique » que du " pouvoir social ". Il appelle aussi " monopole de la compétence scientifique » ce qu'il entend comme " capacité de parler et d'agir légitimement (c'est-à-dire de manière autorisée et avec autorité) en matière de science [...] » (Bourdieu, 1976, 89). Cette composition révèle les couches de l'autorité scientifique qui s'appuie sur l'autorité épistémique. Derrière l'expertise dans un domaine de savoir se profile potentiellement le " pouvoir social » qui en découle, bien que dans certains cas, on peut se demander si ce n'est pas le processus inverse qui est en jeu : le « pouvoir social » serait alors le moteur pour l'acquisition de l'expertise.

Dans le champ scientifique, l'attribution d'autorité se réalise par transmission et par échange de reconnaissance - auxquelles Bourdieu rattache les stratégies de succession. Dans les stratégies de subversion, l'autorité est attribuée à l'issue de conflits portant sur des ruptures conceptuelles : la légitimité acquise autorisant l'accumulation d'un nouveau capital scientifique. II existe une nécessité d'intégration des apports des "dominants " pour rester dans la course : «[...] le champ scientifique doit, entre autres choses, sa spécificité au fait que les concurrents ne peuvent pas se contenter de se distinguer de leurs devanciers déjà reconnus, mais sont contraints, sous peine d'être dépassés et 
'déclassés', d'intégrer leurs acquis dans la construction distincte et distinctive qui les dépasse » (Bourdieu, 1976, 91). Les rapports de force ont donc pour objectif pour les uns de conserver les moyens de production et de reproduction propres au champ alors que pour les autres, il s'agit de succéder aux dominants ou bien de transformer les règles de fonctionnement spécifiques au champ.

L'enjeu final pour le scientifique est d'obtenir la maîtrise des mécanismes constitutifs de son champ : "La lutte dans laquelle chacun des agents doit s'engager pour imposer la valeur de ses produits et sa propre autorité de producteur légitime a toujours en fait pour enjeu le pouvoir d'imposer la définition de la science (i.e. la délimitation du champ des problèmes, des méthodes et des théories qui peuvent être considérés comme scientifiques) [...] " (Bourdieu, 1976, 91). Cette quête repose sur une distribution inégale des places et une dualité dont il est difficile de s'extraire : l'intérêt pour la construction des connaissances d'une discipline scientifique n'est jamais séparé de l'intérêt pour I'autorité scientifique que P. Bourdieu ramène au prestige, à la reconnaissance, à la célébrité... Cette réduction de l'autorité à celle de la récompense symbolique menant à la satisfaction de l'ego fait l'impasse sur l'autorité épistémique; mais elle reste indispensable, car elle fait apparaitre les "bénéfices secondaires " qui se présentent à l'état latent et se tiennent en arrière-plan des discours de rationalité scientifique.

\section{2.}

\section{La citation comme élément de repérage des autorités épistémique et scientifique}

L'activité qui consiste à citer des auteurs dans les articles scientifiques, selon des formules normalisées, est révélatrice de l'attribution d'autorité. Elle intervient dans la restitution écrite des travaux au moment où sont posées les bases destinées à recevoir les nouveaux apports, où sont réalisés les états de l'art indispensables aux constructions théoriques ou à l'établissement des preuves.

\subsection{Le fait citationnel comme élément fondateur de la scientométrie}

L'autorité institutionnelle de l'éditeur s'est construite dès les premières

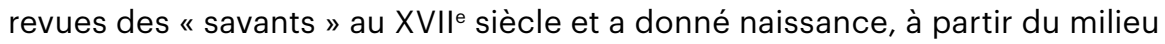
du XIXe siècle, au fur et à mesure du développement capitalistique, à une véritable industrie de la citation orchestrée aujourd'hui encore par les Editeurs possédant les revues phares des domaines scientifiques.

C'est en considérant la « citation non critique de données frauduleuses, incomplètes ou obsolètes » qu'introduit Eugène Garfield en 1955 son article intitulé "Citation indexes for Science » et sous-titré " Une nouvelle dimension à la documentation avec l'association des idées ». Un index dédié aux citations scientifiques est créé qui relie pour la première fois les citations aux docu- 
ments qui les contiennent de manière à faciliter la recherche d'informations. L'objectif est de faciliter le travail de ceux qui cherchent à vérifier les assertions des énoncés, rendre la généalogie des citations explorable et proposer de nouvelles pistes à parcourir (Garfield, 1955). Ce nouvel outil bibliographique basé sur l'indexation associative apporte une évaluation quantitative car il se propose de réserver une entrée qui fait le compte de citations d'un article pour une revue et sur une période donnée; ce qui « peut aider l'historien à mesurer l'influence de l'article ». Tel est le facteur d'impact (IF) défini par Garfield (1955) qui sert donc à mesurer la visibilité des revues scientifiques en établissant des moyennes de citations de chaque article pour chaque revue, afin d'opérer une sélection des « meilleures » revues. En 1963, Eugène Garfield revient sur cette notion d'impact en précisant : " Impact is not the same as importance or significance » (Garfield, 1963). Il se distancie ainsi d'une tendance qui voudrait utiliser les index de citations - initialement prévues pour faciliter la dissémination et la recherche de références - à des fins d'“ évaluations sociologiques ", et s'en servir pour "sélectionner du personnel et des collègues ». Garfield rappelle qu'il n'existe pas de corrélation entre le nombre d'articles publiés par un auteur et la qualité ou l'importance de son travail, même s'il reconnaît l'effet Matthieu. Mis en évidence par Merton (1968) - et théorisé par Price sous le terme de "processus d'avantages cumulés » (Polanco, 1995), l'effet Matthieu se vérifie par le nombre élevé de publications produites par les scientifiques de haut niveau.

Dans un contexte d'augmentation exponentielle de la production scientifique écrite, la scientométrie s'est construite sur l'IF même si son créateur n'imaginait pas le rôle qu'il allait jouer dans l'établissement d'une nouvelle activité scientifique et des usages qui se sont développés, notamment dans l'évaluation du travail de recherche.

En 1965, paraît le premier article utilisant l'index de 1961 de Garfield, par Derek de Solla Price, intitulé "Networks of Scientific Papers " et sous-titré "The pattern of bibliographic references indicates the nature of the scientific research front " dont la conclusion envisage une cartographie des sciences. Il différencie les citations des articles référencés et observe l'évolution de leur taux de citation dans le temps pour en repérer la répétition de motifs (patterns). Est ainsi distingué un "fonds » d'articles " anciens » d'un " front » d'articles récents correspondant à la recherche émergente en physique qui était le corpus principalement étudié dans l'article. Leur distribution topographique projetée sur une carte est calculée avec leur degré de centralité stratégique dans le rang occupé. On est passé de la structure de données sous forme de listes à celles des arborescences et des matrices pour représenter les connaissances scientifiques liées entre elles par des citations.

En 1978, la revue Scientometrics, copubliée par Elsevier Science et Akadémiai Kiadó est fondée. Une conférence est organisée tous les deux ans depuis 1987 par la société savante ISSI (International Society for Scientome- 
trics and Infometrics). Un champ de recherche s'est développé à partir de la représentation de la littérature scientifique par ses réseaux de citations avec I'indicateur mesurable de l'influence. Comme l'indique aujourd'hui le site d'Elsevier, la revue s'intéresse aux caractéristiques quantitatives de la science, de préférence à l'aide d'investigations dans lesquelles le développement et le " mécanisme de la recherche scientifique " sont étudiés par des " méthodes statistiques mathématiques".

Polanco (1995) qui a traduit et synthétisé les apports de Derek de Solla Price souligne la tournure relationnelle que prend l'analyse des citations. Dans cette approche analytique, le caractère social est décelable par les liens mis en évidence entre les acteurs (revues, auteurs, références et citations). Price distingue deux classes de citation : l'« archive " correspond aux références à la littérature « ancienne » et est la connaissance acquise pouvant être représentée par des systèmes de classement ; le " front de recherche " qui correspond à la littérature « récente » est actif et mouvant et peut être cartographié.

Les repérages de patterns, la notion de centralité sont convoqués avec la théorie des graphes pour représenter les relations structurelles des références et citations, sans pourtant s'intéresser aux connaissances qu'elles transmettent. Les projections graphiques ouvrent cependant la voie aux premières représentations cartographiques. Polanco qualifie cette approche scientométrique d'externaliste pour la distinguer de l'approche internaliste de la méthode des mots-associés, née en France au début des années 1980, qui va justement utiliser les mots-clés comme des indicateurs de connaissance (Courtial et al., 1984). L'information bibliographique n'est plus classée par ses citations mais par clustérisation sémantique découvrant la proximité entre documents grâce aux co-occurrences de mots-clés (Grivel, François, 1995).

Considéré comme un critère d'évaluation par les financeurs de la recherche, I'IF sert toujours à classer les revues qui en deviennent très attractives car les retombées en termes de notoriété et d'évaluation seront positives pour les laboratoires dont les chercheurs publiants sont issus. Ceci provoque une course à la publication et le découragement des réviseurs (reviewers) car les auteurs qui voient refoulés leurs articles les re-proposent en moyenne cinq fois avant qu'une revue n'accepte finalement de les publier (Cochard, Delzon, 2015) : ce qui provoque un engorgement et des dysfonctionnements relevés par ces auteurs qui n'hésitent pas à proposer le remplacement de I'IF5 par celui de Google Scholar favorisant le H5 des revues.

\subsection{La citation examinée dans le champ des SIC selon l'approche Bourdieu}

Dans le champ des SIC, Olivesi (2007) a réalisé une étude de l'activité citationnelle d'un congrès de la SFSIC, en tant que traces de la répartition de capital symbolique, et en mettant en évidence ses différentes formes de matérialisation. 
Au sein du champ, il qualifie de « registre primaire » la stratégie citationnelle des agents appartenant à la même discipline. Sont passées au crible les logiques de cooptation, d'allégeance et déférence, de reconnaissance, d'interdépendance, de connivence, d'appartenance, d'autopromotion qui caractérisent le positionnement social dans le champ.

Hors champ, appartiennent au " registre secondaire " les citations d'auteurs pour lesquels il n'existe pas de relations directes mais qui sont convoquées pour divers objectifs. Les quatre catégories concernées sont le placement, l'imposition définitionnelle, la monstration de ressources et les logiques de légitimation.

Le " registre négatif " repose sur la négation " réelle ou euphémisée » de la relation des agents et de ses termes et comprend six catégories qui vont servir à rendre visible ou invisible les agents : I'occultation ou la négation symbolique (concerne le registre primaire), la raréfaction, l'ignorance (négation des logiques du registre secondaire), la critique, l'allusion.

Cette triple typologie est intéressante car elle met en lumière les jeux relationnels stratégiques qui s'établissent à l'occasion de l'adoption ou du rejet de concepts par les communautés. Elle fait de plus entrer dans le jeu relationnel la non-citation qui repose sur une stratégie d'évitement et d'écartement des auteurs indésirables. Pour les nouveaux entrants, il s'agira « avant tout de montrer que l'on sait goûter, c'est-à-dire discriminer ceux qu'il s'agit de citer et ceux qu'il faut éviter de citer. Savoir 'bien' citer conditionne le fait d'être reconnu comme légitime et d'être intégré dans le cénacle de ceux qui font le bon goût et qui le partagent »(Olivesi, 2007, 87).

Pourtant cette diversité de l'arrière-plan de l'activité citationnelle mise en évidence ici par et pour les SIC, qui devrait mener à une prise de conscience des contraintes et à une déprise des croyances, n'apparaît pas avoir été prise en compte par les systèmes d'évaluation du mérite des chercheurs, et en premier lieu par les « infrastructures informationnelles » (Pontille et Torny, 2013) basées sur le comptage des citations.

Notons ici deux caractéristiques relevées dans diverses disciplines : d'une part, la publication dans une revue est souvent liée aux réseaux interpersonnels entretenus avec le comité de rédaction, d'autre part l'évaluation du degré de prestige d'une revue est subjective.

\subsection{La compréhension de l'activité citationnelle}

En 1984, paraît le premier ouvrage étudiant le processus social de la citation (Cronin, 1984). Il se compose essentiellement d'une compilation d'analyses et d'approches classificatoires issues principalement d'historiens et sociologues des sciences. Ayant implicitement intégré le travail de Pierre Bourdieu cité en bibliographie, l'introduction souligne le processus social de la science et la 
dépendance des actions et comportements des scientifiques aux contextes de l'activité professionnelle et disciplinaire.

A la recherche d'une théorie de la citation, Cronin fait le tour de la littérature s'étant penchée sur la question mais se trouve dans l'impossibilité d'en synthétiser les différentes analyses compte-tenu de la grande diversité des approches disciplinaires.

Les fonctions du système de communication scientifique y sont rappelées : disséminer les savoirs, assurer la préservation des standards, distribuer les crédits et la reconnaissance à ceux dont le travail a contribué au développement des idées dans différents champs (Cronin, 1984).

Le contexte de l'écriture de cet ouvrage est qu'en vingt ans l'accès aux index de citations s'est diversifié : création en 1964 du Scientific citation index (SCI), en 1972 du Social science citation index (SSCI), en 1975 du Journal of citations report (JCR). Et l'on commence à s'intéresser à la pratique citationnelle qui auparavant n'attirait l'attention qu'en cas de litige ou de manquement grave à l'étiquette (Cronin, 1984, 7).

Est alors mis en cause le système normatif de la science. De grands principes sont censés guider les pratiques scientifiques et Mitroff (1974) - cité par Cronin (1984) - relève onze normes en science dans l'objectif de les discuter et de restituer la subjectivité qui en est éliminée. Parmi-celles-ci, notons la foi dans la rationalité et la neutralité émotionnelle (qui est la condition pour que le principe précédent fonctionne), mais aussi l'impartialité : un scientifique serait concerné uniquement par la production d'un nouveau savoir et non les conséquences de ses usages. On peut observer que ces grands principes sont essentiellement moraux tout en se distinguant de la vie en société régulée par le droit : les principes de "loyauté » et de "liberté " procèdent d'une double isolation : la production du scientifique s'effectue au sein d'une communauté à laquelle il doit rester fidèle tout en résistant à l'environnement susceptible d'en limiter l'action.

Dans l'objectif de cerner les raisons pour lesquelles les auteurs citent les travaux des autres, il reprend la liste de Weinstock (1971) qui a entrepris de classer quinze fonctions de citations qualifiées de sérieuses, dans le but d'attribuer des motivations. On y voit que sont mélangées autorité scientifique (rendre hommage aux pionniers, aider à diffuser des travaux peu disséminés, pauvrement indexés ou non cités) et autorité épistémique (authentifier les données et classes de faits, etc.). 


\begin{tabular}{|c|c|}
\hline $\begin{array}{l}\text { Groupe } 1 \text { : contribution scientifique } \\
\text { originale ou intention de l'article ci- } \\
\text { tant }\end{array}$ & 14) résumé ou condensé \\
\hline 1) décrit le phénomène observé & 15) erratum \\
\hline 2) transforme les données & 16) suite \\
\hline 3) explication & $\begin{array}{l}\text { 17) précurseur (réfère à une publica- } \\
\text { tion à venir) }\end{array}$ \\
\hline 4) hypothèse ou théorie & $\begin{array}{l}\text { 18) inclusion (la publication citante } \\
\text { contient le papier cité) }\end{array}$ \\
\hline \multicolumn{2}{|l|}{ 5) calcul à partir d'une théorie } \\
\hline 6) prédiction & $\begin{array}{l}\text { Groupe } 4 \text { : caractère de la contribu- } \\
\text { tion scientifique du papier cité dans } \\
\text { le papier citant }\end{array}$ \\
\hline 7) définition ou notation & 19) seulement noté \\
\hline \multirow[t]{2}{*}{ 8) état d'une technique expérimentale } & 20) distingué (non pertinent) \\
\hline & 21) critiqué ou comparé \\
\hline $\begin{array}{l}\text { Groupe } 2 \text { : contribution du papier ci- } \\
\text { tant autre que contribution scienti- } \\
\text { fique originale }\end{array}$ & 22) appliqué \\
\hline 9) article de synthèse & 23) amélioré ou modifié \\
\hline 10) bibliographie & 24) remplacé \\
\hline \multirow[t]{2}{*}{ 11) données cumulées } & $\begin{array}{l}\text { 25) change la précision du travail cité } \\
\text { (+ ou -) }\end{array}$ \\
\hline & $\begin{array}{l}\text { 26) change l'échelle d'application (+ } \\
\text { ou -) }\end{array}$ \\
\hline $\begin{array}{l}\text { Groupe } 3 \text { : identité ou continuité re- } \\
\text { lationnelle du papier citant au papier } \\
\text { cité }\end{array}$ & 27) questionné (doute exprimé) \\
\hline 12) un ou deux auteurs en commun & 28) affirmé \\
\hline 13) même texte (versions & 29) réfuté \\
\hline
\end{tabular}

Tableau 1 : Relations de citation en littérature scientifique (Lipetz, 1965) 


\section{A. documentation des sources primaires - références à des textes littéraires, lettres, etc.}

1) appuyer une opinion ou une affirmation factuelle sur l'auteur ou le travail spécifique discuté dans le travail citant

2) appuyer un point de vue en dehors du thème central du travail citant

3) appuyer une affirmation factuelle en dehors du thème central du travail citant

\section{B. Documentation des sources secondaires - références à des travaux antérieurs}

reconnaître le travail pionnier d'autres scientifiques,

1) Indépendant d'une approbation ou d'une désapprobation de l'auteur citant indiquer l'état de la recherche présente, l'ensemble des points de vues, ou les opinions dominantes sur une thématique,

discuter du sens d'un terme ou référer à un travail dans lequel un terme donné ou un symbole apparaît pour la première fois,

soutenir un point de vue de l'auteur citant,

2) Représentant une approbation de l'auteur cité

soutenir une affirmation factuelle de l'auteur citant,

faire progresser une idée,

reconnaître une dette intellectuelle,

exprimer un désaccord avec le point de vue du chercheur cité,

3) Représente une désapprobation de l'auteur citant

exprimer un désaccord avec une affirmation factuelle du chercheur cité,

exprimer un point de vue mixte

\section{Documentation des sources primaires ou secondaires}

Orienter vers une lecture complémentaire

Indiquer une référence bibliographique d'une édition spécifique

Tableau 2 : Grille d'identification catégorielle des citations (Frost, 1979) 
Cronin (1984) remarque cependant la difficulté d'attribuer des motivations à des citations qui, en apparence, représentent des explications plausibles mais n'apportent rien sur les intentions de leurs auteurs. II s'intéresse alors à l'analyse de contenus et des contextes et reprend les travaux de Lipetz (1965) qui, dans l'objectif d'améliorer la qualité des résultats de la recherche dans les bases de citations, suggère d'ajouter des indicateurs relationnels qui décrivent pleinement la fonction ou le caractère de chaque citation. Quatre groupes avaient été identifiés à partir d'un corpus de citations en physique (Tableau 1).

Les différents types de citation correspondant à cette grille interprétative étaient encodés et entraient dans l'indexation des articles, ce qui devait permettre d'identifier plus rapidement les apports d'un article par rapport à celui cité.

Un nouveau projet envisageant les citations comme des opérateurs relationnels est repris et testé dans les humanités dans l'objectif de détecter les tendances à s'appuyer sur des preuves non empiriques ou circonstancielles. Carolyn Frost (1979) établit une liste de classifiants de citation testée sur un corpus de littérature allemande issu d'un travail réalisé pendant sa thèse.

En dépit du nombre d'études réalisées sur l'activité de citation, E. Garfield (1996) reconnaît deux faits : l'absence d'enseignement dédié à cette pratique d'écriture qui relève d'une pratique ancienne d'imitation ; la focalisation sur l'instrument d'évaluation que sont devenus les index de citations au détriment de leur visée première : I'amélioration de la recherche d'informations.

En 1998, une nouvelle fois le constat est fait du manque d'une théorie complète de la citation malgré les multiples propositions réalisées selon différentes perspectives théoriques. Leydesdorff (1998) reconnaît le processus social de la citation, ce qui questionne les conditions sous lesquelles elle peut être utilisée pour des mesures d'impact, de qualité, de pertinence, de réputation ; un besoin d'analyse méta-théorique est identifié. L'analyse de citation utilise les paires cité-citant comme des relations. II s'agit donc d'étudier les traces d'un système réseau multidimensionnel dont les distributions indiquent des frontières disciplinaires mouvantes.

Ces quelques exemples sont indicateurs de la difficulté à analyser l'activité citationnelle qui superposent et quelquefois mélangent l'autorité épistémique qui se réfère au texte repérable par son insertion dans une démonstration ou une argumentation et l'autorité scientifique qui se réfère aux auteurs citant et cités et dont une partie sert à conforter ou à créer les places des agents dans leur champ.

\subsection{Le futur de la citation mesurée et normalisée}

Alors que l'activité citationnelle est relativement récente puisqu'elle apparaît dans le milieu scientifique au milieu du XIX ${ }^{e}$ siècle, en quinze ans l'évaluation s'est déplacée de la revue vers l'article puis vers l'auteur. Sans entrer dans 
l'exhaustivité des algorithmes développés, remarquons l'évolution suivante dans les métriques du facteur d'impact.

A l'origine, I'IF créé par Garfield (1972) dans les années 1960 concerne les revues (Journal Impact Factor) et a pour objectif le repérage des revues les plus pertinentes d'une discipline, il s'agit de mesurer l'importance d'une revue. L'Eigenfactor (EF) est un Facteur d'influence proposant de classer les revues d'une manière similaire à celle de l'algorithme de classement (ranking) de Google. Il s'agit d'un indicateur prenant en compte l'origine des citations (c'est-à-dire l'article) et leur prestige défini par le nombre de citations reçues. L'Article influence (AI) lié au précédent détermine l'influence moyenne de chaque article sur cinq ans, indépendamment de la taille de la revue. L'indice de Hirsch introduit en 2005 ( $\mathrm{H}$-index), initialement prévu pour évaluer la notoriété d'un auteur, a aussi pour objectif de classer les revues par langue, par catégorie et sous-catégorie. Son intégration rapide dans les algorithmes - Scopus d'Elsevier et Web of Science (WoS) de Thomson Reuters - fait dire à Pontille et Torny (2013) que la mesure individuelle est devenue un sujet de recherche central dans la production d'algorithmes pour les bibliomètres. Dans le même temps, les outils bibliométriques deviennent accessibles à chaque chercheur (Pontille et Torny, 2013). Notons les modèles alternatifs comme celui de Citebase, outil d'évaluation des citations provenant du réservoir pionnier ArXiv. L'algorithme de citations développé par Harnad et Brody repose sur l'interrogation de la base contenant principalement des archives en mathématiques, informatique, astrophysique et en biologie quantitative. Sont comptabilisés et mis en rapport le nombre d'ouvertures de fichiers correspondant au texte intégral d'un article et celui des citations y faisant référence dans d'autres articles (Durand-Barthez, 2008). Google Scholar, qui s'apparie à l'algorithme de Hirsch, est devenu un outil massivement utilisé vers lequel pointent différents éditeurs scientifiques ; ceci est représentatif du glissement qui s'est opéré de l'autorité de la revue vers la notoriété de l'article.

En 2012, Lozano, Larivière et Gingras publient les résultats d'une étude menée sur la totalité des sciences naturelles, médicales et sociales du WoS, indiquant un affaiblissement de I'IF depuis l'introduction des revues électroniques dans les années 1990 : la proportion d'articles très fréquemment cités ne provenant pas des revues les plus citées continue de s'accroître. En 2016, un nouvel article de Larivière et al. confirme cette tendance et démontre que moins d'un article sur quatre obtient la valeur moyenne et se situe donc sous le facteur d'impact pour onze revues multidisciplinaires qualifiées de très importantes. Cet affaiblissement de la mesure par le « facteur d'impact " pourrait signifier le basculement - à terme - d'un système vers d'autres modes d'évaluation.

Rappelons que l'évaluation de la recherche à l'aide d'indicateurs statistiques a toujours été critiquée (Gingras, 2008) ; elle empêche à terme la production de savoirs par absence de prise de risques et volonté de se conformer à une norme (Revue Cités, 2009). Ceci d'autant plus qu'aucune vérification des 
citations n'est réalisée et que les citations tronquées ou inadaptées trahissant la non lecture des articles référencés sont régulièrement pointées.

Pour donner du sens aux citations dans leur analyse quantitative, la prise en compte du contexte de leur matérialisation par les algorithmes est recherchée depuis plus d'une dizaine d'années. Récemment, le Hip-Index (Influence-primed Index) vise à capturer le contexte de la citation afin de lui affecter un poids : plus que le nombre de citations obtenues par une référence, c'est le nombre de fois où un auteur est cité dans un même article qui va discriminer les citations (Zhu et al., 2015). De même pour Kazi et al. (2016) qui ont mis au point un algorithme analysant la tonalité positive ou négative des citations, détectant les auto-références et les similarités sémantiques entre l'article citant et l'article cité.

Enfin, les technologies du web sémantique se diffusant lentement mais sûrement dans la sphère de la publication académique, le temps se rapproche pour certaines disciplines où la citation sera normalisée avec un identifiant pérenne.

Dernière innovation en date de cette orientation, le système Dokieli (Capadisli et al., 2015) est un véritable couteau-suisse de la publication scientifique. Il s'agit d'un outil-éditeur d'article, utilisable en local ou sur un serveur, et qui accepte les commentaires des pairs reviewers ou des simples lecteurs. Une grande richesse fonctionnelle caractérise le processus d'édition : les objets de granularité fine de l'article sont sémantiquement décrits et accessibles, comme les concepts, les hypothèses, les arguments. Réalisé en HTML5, un identifiant unique (URI) est généré pour chaque section de l'article, l'auteur étant à même d'encoder chaque concept qu'il juge digne de référencer. Ce qui concrétise la publication scientifique reliée en open linked-data. L'originalité de cet éditeur est aussi de proposer plusieurs formats à la lecture (feuilles de style, pdf, etc.), l'ajout de commentaires, le référencement sémantique et le partage via le web 2.0. Lisibles par les humains et les machines, liables pour leur republication, réutilisables pour leur contenu, interopérables, ces prototypes d'articles contribuent à la formalisation des publications scientifiques selon les normes de disciplines éloignées des sciences humaines et sociales. Cependant, cette innovation sur la sémantique ouvre la voie à un art technique de la citation qui renouvelle sa puissance connective. L'appareillage critique des « humanités numériques » pourrait s'en trouver enrichi. 


\section{3. \\ De la sélection des publications par le tri à la mesure des échanges qu'elles suscitent}

\subsection{La mesure des échanges sur le web}

En une quinzaine d'années un basculement s'est produit : on est passé d'une sélection de l'excellence par réduction de la masse disponible d'informations à la création d'infrastructures informationnelles visant à capturer la totalité des échanges. Ce basculement s'opère à deux niveaux. Les données disponibles se sont massivement multipliées imposant de nouveaux modes informatiques d'exploration; la réception par le lectorat des articles scientifiques circulant sur le web se compose d'une production qui est à même d'être mesurée. Notons ici que les mouvements de l'accès libre aux résultats des recherches et des archives ouvertes qui ont été impulsés et animés par des chercheurs et des professionnels de la documentation scientifique ont joué un rôle non négligeable dans la diversification de l'accès à l'information scientifique. Michael Jensen, directeur de la stratégie de la communication Web aux Presses nationales académiques (Etats-Unis) remarque ce renversement de situation dès 2006. Il faisait le constat que le passage de la rareté de I'information à l'ère de l'abondance allait modifier la façon dont les mesures pourraient être utilisées pour établir l'autorité académique, l'importance et la validité scientifique. Selon cet acteur, le travail de l'éditeur basé sur une gestion de la rareté changeait d'autant plus qu'il n'était plus l'intermédiaire indispensable à la publicisation des contenus.

Jensen (2006) a proposé de quantifier les nouvelles manières de référencer les contenus : traces de lecture (bookmarking, attention révélée par le blogging, etc.) ; calcul de la valeur du réseau à partir de ce que tout l'auteur a atteint, vu et lu; prise en compte des échos donnés aux documents (sélections de "best of ", indexations diverses, etc.) ; analyse de tonalité des commentaires; pourcentage de portions de documents citées par d'autres document. Dans le même temps, il s'intéressait au prestige de l'éditeur, des reviewers, des commentateurs et à la qualité des affiliations institutionnelles de l'auteur. Les " altmetrics » (Mahé, Prime-Claverie, 2016), qui mettent en œuvre les propositions de Jensen, visent à quantifier les usages de la production scientifique via le signalement des références par les logiciels de gestion de références bibliographiques (LGRB), les blogs, les réseaux, les téléchargements, etc.

Le rachat du LGRB Mendeley par Elsevier lui donne directement accès aux pratiques de partage disciplinaires et aux usages des différentes plateformes académiques de diffusion d'articles. Dernièrement, les agrégateurs de revues comme Wiley, ont intégré à la lecture de leurs publications le format PDF enrichi. Sont disponibles, outre les jeux de données ayant servi de matière à la recherche, les références citées dans l'article et la liste des publications citant l'article qui s'affichent dans une colonne supplémentaire. L'intégration des 
fonctionnalités du LGRB Readcube par nombre d'éditeurs indique la connexion systématique de Google Scholar avec les réservoirs de publications des éditeurs (ex : Springer).

Du côté des nouveaux entrants de la communication scientifique, le lancement des réseaux sociaux de chercheurs comme Academia.edu, ResearchGate qui ont pour objectif de capitaliser les informations explicites et implicites obtenues à partir des échanges entre membres-chercheurs, a produit des agrégateurs de $\mathrm{CV}$ de dimension mondiale totalisant près de 30 millions de membres (Bouchard, 2015). Ces plateformes exploitent la volonté des auteurs de se rendre visibles et encouragent fortement les échanges entre membres en valorisant cette activité avec des index de mesure. Pour ResearchGate, le RG Reach est venu compléter le RG Score ${ }^{2}$ après avoir intégré I'H-Index qui détecte l'autocitation. Leur particularité est de moissonner gratuitement les Serveurs d'archives ouvertes et de s'auto-alimenter.

Alors dire que l'activité citationnelle s'est largement développée hors les circuits traditionnels de la communication scientifique apparaît questionnable aujourd'hui. Certes les altmetrics ne différencient pas les types de lecteurs : s'agit-il de lecteurs chercheurs, d'amateurs, de bibliothécaires, de journalistes, de documentalistes ou du "simple » public ? La lecture profane des amateurs a toujours existé, c'est simplement sa dimension qui a changé. Ce que nous avions pris pour des contradictions dont nous nous proposions d'explorer la profondeur apparaît aujourd'hui se situer dans la continuité des travaux liés à l'évaluation quantitative des résultats de la recherche. Une tendance à l'autoréférence est cependant à retenir.

\subsection{L'autoréférence du web social}

A partir d'une recherche sur plus de 90000 articles publiés par la revue PLOS dans sept journaux médicaux, l'impact de la visibilité des réseaux sur les index de citations a été conceptualisé par Ebrahimy et al. (2016). Les actions de téléchargement de fichiers (save), de discussion et de recommandations ont été corrélées avec les citations de Scopus, Web of Science, PubMed Central et CrossRef. Alors que la visibilité est reliée positivement aux actions de sauvegarde de fichiers, la visibilité est reliée négativement aux discussions, et la troisième hypothèse n’a pu être vérifiée.

La " citation 2.0 " se réalise par des services dépendants de systèmes de recommandation et de référencement ; les graphes dirigés de réseautage obéissent à des hiérarchies (Merzeau, 2013) et apparaissent être plus de l'ordre de l'influence choisie que celle de l'autorité. Elles relèvent plutôt des pratiques légitimantes. Un simple coup d'œil aux réseaux agrégés par Twitter permet

2 Le RG Reach mesure la visibilité au sein du réseau tandis que le RG Score mesure l'accès aux publications référencées. 
de le vérifier. L'espace des relations créées par Twitter conjugue des phases autoritatives (devenir auteur sans autorité) quelquefois subversives et donc en rupture avec les "dominants ", avec des phases de transmission de " dominants " vers des cours attentives. Chaque rôle pouvant être endossé selon le contexte de l'écriture. Les phénomènes d'autoréférence et des mécanismes d'autocitation qui sont observables indiquent que de nouvelles règles de présentation se forment et même s'ils sont quelquefois de l'ordre de plaisanteries entre happy few, leur fréquence et leur systématisation donne à penser qu'ils servent aussi à flatter les égos.

Le processus d'évaluation des articles en « double aveugle " s'est récemment imposé aux revues de sciences humaines et sociales, l'objectif étant aussi de préserver des conflits d'intérêts les parties prenantes que sont les proposants et leurs relecteurs. Mais la médiatisation de l'avancement des recherches sur les réseaux sociaux présuppose l'identification des auteurs mais pas l'identification de leurs lecteurs qui n'appartiennent pas toujours au champ scientifique. L'incarnation de l'auteur en ressort renforcée et contribue indirectement à son institutionnalisation (invitations à communiquer, médiatisations diverses). L'auteur incarné par les réseaux s'impose alors aux côtés de l'auteur institutionnalisé par les revues. Cette tendance marque aussi l'obligation pour le chercheur publiant de devoir assurer sa propre publicité pour exister dans l'espace électronique, cette nouvelle charge organisant peut-être aussi une tyrannie de la visibilité.

\section{4. Conclusion}

Dans cet article, en cherchant les modifications à l'œuvre dans l'établissement de l'autorité scientifique, nous avons reconnu sa matérialisation en examinant le rôle central pris par la mesure des citations dans la communication scientifique. Nous remarquons que cette autorité est monnayée par les agents éditeurs, qui attribuent du crédit avec des notions très peu explicitées dans les algorithmes qu'ils utilisent : le prestige et l'influence. Le fait citationnel est révélateur des tensions entre champ social et discipline, entre autorité épistémique et scientifique.

L'appropriation par les chercheurs d'un ensemble de pratiques de diffusion (présentations de conférences, signets partagés, blogging, réseautage social) est le signe d'une mise en commun renouvelée de diverses références. Est alimenté un processus de légitimation qui apparaît extérieur à l'appareillage critique et technique de l'évaluation scientifique. Il s'agit d'un mouvement vers l'autoréférence qui se fond dans la masse informationnelle du web.

Les métriques s'avèrent insuffisantes à situer la qualité des publications. Certes, elles mesurent l'influence mais leurs modalités de déploiement restent 
à détailler. Olivesi a montré l'indéniabilité des relations existantes entre le statut social d'un auteur et ses chances d'être cité : plus que l'activité éditoriale, c'est le rayonnement de l'auteur qui va garantir une visibilité à sa production. L'instrumentalisation des réseaux sociaux pour fabriquer de la notoriété en vue d'exister scientifiquement correspond-elle à un fantasme ou une réalité ? Plus que son autorité épistémique, c'est la réputation de l'auteur qui entre ici en jeu.

Enfin, alors que l'art de la citation n'est pas enseigné et que cette pratique recouvre de multiples motivations pouvant être éloignées de leur rôle épistémique, leur technicisation normalisée pourrait éclaircir leur rôle, mais aussi constituer une forme de contrainte discriminante qui ne conviendra pas à toutes les disciplines scientifiques. 
Bibliographie

Bachelard G. (2013 [1934]). Le nouvel esprit scientifique, Paris, Presses Universitaires de France.

Bouchard A. (2015). "Où en est-on des réseaux sociaux académiques ? ", Billet de Blog du 15 mai 2015. Disponible sur http:// urfistinfo.hypotheses.org/2896 (page consultée le 12 février 2017).

Bourdieu P. (1976). « Le champ scientifique ". In Actes de la recherche en sciences sociales, vol. 2, n²-3, juin 1976, "La production de l'idéologie dominante ", p. 88-104. Disponible sur http:// www.persee.fr/doc/arss_03355322_1976_num_2_2_3454 (page consultée le 12 février 2017).

Cités. (2009/1). « L'idéologie de l'évaluation. La grande imposture ", $n^{\circ} 37$. 192 p. Disponible sur http://www. cairn.info/revue-cites-2009-1.htm (page consultée le 12 février 2017).

Cochard H., Delzon S. (2015). "L'édition scientifique doit faire sa révolution et mettre à l'index ses indices ». In Publier la science, Juin.

Courtial J. P., Callon M., Sigogneau M. (1984). «Is indexing trustworthy? Classification of articles through co-word analysis ". In Information Scientist, vol. 9, n², p. 47-56.

Cronin B. (1984). The citation process: the role and significance of citations in scientific communication, Londres, Taylor Graham.

Durand-Barthez M. (2008). « Historique et critique du facteur d'impact ", Collection Schedae, Presses Universitaires de Caen, vol. 7, n¹, p. 67-76. Disponible sur https://www.unicaen.fr/puc/ images/preprint0072008.pdf (page consultée le 12 février 2017).

Ebrahimy S., Mehrad J., Setareh F. et al. (2016). " Path analysis of the relationship between visibility and citation: the mediating roles of save, discussion, and recommendation metrics ". In Scientometrics, $n^{\circ} 109$, p. 1497-1510.

Fleck L. (2005 [1935]). Genèse et développement d'un fait scientifique, Paris, Les Belles Lettres.

Frost C. (1979). « The use of citations in literary research: a preliminary classification of citation functions ". In The Library Quarterly: Information, Community, Policy, vol. 49, n 4, p. 399-414.

Garfield E. (1955). " Citation indexes for science: A new dimension in documentation through association of ideas ". In Science, vol. 122, $n^{\circ} 3159$, p. 108-11, July.

Garfield E. (1963). « Citation indexes in sociological and historical research ". In American Documentation, vol. 14, n 4, p. 289-91, October.

Garfield E. (1996). « When to Cite ». In Library Quarterly, vol. $66, n^{\circ} 4$, p. 449-458. 
Jensen M. (2007). « The New Metrics of Scholarly Authority ". In The Chronicle Review, $n^{\circ} 53$, p. 41. Disponible sur http://chronicle.com/article/ The-New-Metrics-of-Scholarly/5449 (page consultée le 12 février 2017).

Gingras Y. (2008). « La Fièvre de l'évaluation de la recherche du mauvais usage de faux indicateurs ". In Revue d'histoire moderne et contemporaine, vol. 5 , $n^{\circ} 4$ bis, p. 67-79.

Kazi P., Patwardhan M., Joglekar P. (2016). « Towards a new perspective on context based citation index of research articles $"$. In Scientometrics, $n^{\circ} 107$, p. 103-121.

Nordenstreng K. (2007). « Discipline or field? Soul-searching in communication research ». In Nordicom Review, $\mathrm{n}^{\circ}$ 28: Jubilee Issue, p. 211-222, Nordicom.

Kuhn T. (2008 [1962]). La structure des révolutions scientifiques, Paris, Flammarion.

Lahire B. (2001). «1. Champ, horschamp, contrechamp ». In Le travail sociologique de Pierre Bourdieu (Lahire dir.), La Découverte, p. 23-57. Disponible sur http://www.cairn.info/ le-travail-sociologique-de-pierrebourdieu--2707130869-page-23.htm (page consultée le 12 février 2017).

Larivière V. et al. (2016). « A simple proposal for the publication of journal citation distributions ». Disponible sur http://www. biorxiv.org/content/biorxiv/ early/2016/07/05/062109.full.pdf (page consultée le 12 février 2017).
Latour B., Woolgar S. (1988). La vie de laboratoire. La production des faits scientifiques, Paris, La Découverte.

Lefebvre M. (2008). « L'évaluation des savoirs scientifiques : modalités et enjeux ». In La publication scientifique : analyses et perspectives, Hermès, p. 299-316.

Lipetz B.-A. (1965). « Improvement of the selectivity of citation indexes to science literature through inclusion of citation relationship indicator ». In American Documentation, $\mathrm{n}^{\circ} 16$, p. 81-90.

Lozano G. A., Larivière V., Gingras Y. (2012). "The weakening relationship between the impact factor and papers citations in the digital age ». In Journal of the American Society for Information Science and Technology, n63, p. 2140-2145.

Mahé A., Prime-Claverie C. (2016). «Altmetrics - Nouvelles mesures de la visibilité des résultats de la recherche - Des métriques traditionnelles de la science aux altmetrics ». In Techniques de l'ingénieur, "Documents numériques : diffusion et recherche d'information ». Disponible sur http://www.techniques-ingenieur.fr/base-documentaire/ technologies-de-l-information-th9/ documents-numeriques-diffusion-et-recherche-d-information-42482210/altmetrics-h7730/ (page consultée le 12 février 2017).

Merton R.K. (1968). "The Matthew effect in science ". Science, $n^{\circ} 159$, p. 56-63. 
Merzeau L. (2013). « Twitter, une machine à fabriquer de l'autorité ? ". In Pelissier N., Gallezot G. (dir.), Twitter : un monde en tout petit, Paris, L'Harmattan, p. 35-51.

Olivesi S. (2007). Référence, déférence. Une sociologie de la citation, Paris, L'Harmattan.

Origgi G. (2013). « Un certain regard. Pour une épistémologie de la réputation ". In Communications, n 93, p. 101-120. Disponible sur www.cairn.info/revue-communications-2013-2-page-101.htm (page consultée le 12 février 2017).

Polanco X. (1995). «Aux sources de la scientométrie ». In Solaris, $n^{\circ} 2$, Dossier « Bibliométrie, Scientométrie, Infométrie ». Disponible sur http://gabriel.gallezot.free.fr/ Solaris/d02/2polanco1.html (page consultée le 12 février 2017).

Pontille D. (2006). «Qu'est-ce qu'un auteur scientifique? ». In Sciences de la Société, n 67, p. 77-93.

Pontille D., Torny D. (2010). « Revues qui comptent, revues qu'on compte : produire des classements en économie et gestion ". In Revue de la régulation, $\mathrm{n}^{\circ} 8$. Disponible sur http://regulation.revues.org/8881 (page consultée le 12 février 2017).
Pontille D., Torny D. (2013). « La manufacture de l'évaluation scientifique. Algorithmes, jeux de données et outils bibliométriques ". In Réseaux, n²17, p. 23-61.

Disponible sur www.cairn.info/ revue-reseaux-2013-1-page-23.htm (page consultée le 12 février 2017).

Valenzuela M., Ha V., Etzioni O. (2015). « Identifying Meaningful Citations ". In AAAI Workshop - Technical Report, vol. WS-15-13, p. 21-26.

Weinstock M. (1971). « Citation indexes ». In Encyclopaedia of Library \& Information Science, $n^{\circ}$ 5, p. 1640.

Zhu X., Turney P., Lemire D., Vellino A. (2015). « Measuring academic influence: Not all citations are equal ». In Journal of the Association for Information Science and Technology, n66, p. 408-427. 